\title{
A new theory of light and matter
}

\author{
John Williamson* \\ University of Glasgow \\ E-mail: john.williamsoneglasgow.ac.uk
}

\begin{abstract}
A new, linear field theory of light and matter is proposed, sharpening the principle of relativity and adding four new coupled differential equations to the original four of Maxwell. The new theory, though continuous, allows only propagating pure field solutions with an integral angular momentum. These are identified with the photon. Solutions with rest-mass are necessarily charged, with a value close to the elementary charge [1]. They have a double-covering rotation in momentum space with a resulting half-integral spin and statistics, as was discussed on the basis of an earlier non-linear theory [2]. The result is that, just as was the case for de Broglie's concept of the harmony of phases [3] which led to his famous relation and the subsequent development of quantum mechanics, it is a rigorous consideration of the principle of relativity that leads to the quantisation of light and matter.
\end{abstract}

References

[1] Williamson, J.G. \& van der Mark, M.B. Is the electron a photon with toroidal topology?, Ann. Fondation L. de Broglie 22, 133 (1997).

[2] Williamson, J.G. Fermions from Bosons and the origin of the exclusion principle, Proceedings of MENDEL 2012.

[3] de Broglie, L. Recherches sur la théorie des quanta, Ann. Phys. Ser. 10 3, 22 (1925).

Frontiers of Fundamental Physics 14 - FFP14,

15-18 July 2014

Aix Marseille University (AMU) Saint-Charles Campus, Marseille

${ }^{*}$ Speaker. 